\title{
Animations Analysis in Experimental Fluid Dynamics
}

\author{
I. A. Znamenskaya ${ }^{1}$, N. N. Sysoev², I. A. Doroshchenko3 \\ Faculty of Physics, Lomonosov Moscow State University \\ 1 ORCID: oooo-ooo1-6362-9496, znamen@phys.msu.ru \\ 2 ORCID: 0000-0002-1162-7680 \\ 3 ORCID: 0000-0002-0488-0020, doroshenko.igor@physics.msu.ru
}

\begin{abstract}
Digital imaging became one of the main tools for studying fluid dynamics. Modern highspeed cameras support video recording at high frame rates which allows studying of the extended high-speed processes. We demonstrate here different animations based on various methods of visualization, such as water temperature evolution with a frame rate of $115 \mathrm{~Hz}$; high-speed shadowgraph visualization of different flows - supersonic water jet formation process (100 ooo frames/s), shadowgraph animations of the shock waves created by the pulsed discharges (124 000 frames/s). Also, as an example of the plasma radiation emission technique, we offer 9 sequential images of the pulse gas discharge visualization obtained by the high-speed CCD camera with the 100 ns delay between frames. We used a commercial and developed in-house software based on machine vision and learning techniques for automatic flow animations processing. The examples of the automatic bow shock and oblique shock detection using Canny edge detection and Hough transform on the pre-trained convolutional neural network are provided and discussed.

Keywords: Flow visualization, thermographic animation, high-speed shadowgraph animation, discharge flow visualization, digital image processing, convolutional neural networks, edge detection, Hough transform.
\end{abstract}

\section{Introduction}

Non-stationary processes in gas, liquids, plasmas, multiphase media are recorded with specialised equipment, respectively starting from direct observation with the eyes, then observation using pinhole cameras, glass optics [1,2]. Visualization of flows' parameters is based on the physical properties of electromagnetic radiation and its interactions with media, including physical processes:

- Scattering

- Refraction

- Absorption

- Reflection

- Interference

- Dispersion

- Luminescence

- Emission

From the middle of the 19th century, photographic equipment began to be used for scientific visualization. French naturalist J. E. Marey was the first to use multi-frame photography to record flow movements. His chronophotographic gun (1882) was capable to record 12 consecutive frames per second. In the wind tunnel he visualized flow around obstacles and the transition to turbulence using a smoke system. Later film cameras, video cameras, and high-speed drum cameras were used. In the middle of the 2oth century, electrooptical devices appeared. Modern digital high-speed cameras can record quite fast processes in fluids with rate up to 1 million frames/s. For capturing films of non-stationary flows the 
visualisation equipment should have exposure time less then minimal flow characteristic time; thus is the time interval between 2 frames.

Obtaining visual information about flows (fluid dynamic processes) is the creation of experimental databases for verification of computer fluids dynamics (CFD) software. The challange of the CFD codes verification is matching of experimental and calculated visualization data - flow images. $3 \mathrm{D}$ and $4 \mathrm{D}$ data (animations) are problematic for this matching. So 2D images extracted from animations should be used. Big number of consequent images need a special modern approach for being matched. The use of machine learning is the quite promising approach to experimental digital animation analyses in nonstationary fluid dynamics.

\section{Thermographic animation}

The slow motion of liquids may be recorded with digital cameras at 10000 frames per second with sufficient space resolution. Modern thermal imaging cameras resolution is considerably lower than that of optical cameras. The frame rate is less than 1000 frames/s.

Fig. 1 presents thermographic image taken from video film of turbulent boundary water flow at $115 \mathrm{~Hz}$ - submerged impact hot jet in cold water (false colors). Being recorded through an IR-transparent window, thermal imager visualizes instant images of infrared radiation from a thin near-surface water boundary layer $[3,4]$. The time dependent curves of the measured thermal time-resolved signals at four points of flow field are obtained using FLIR software (Fig. 1).
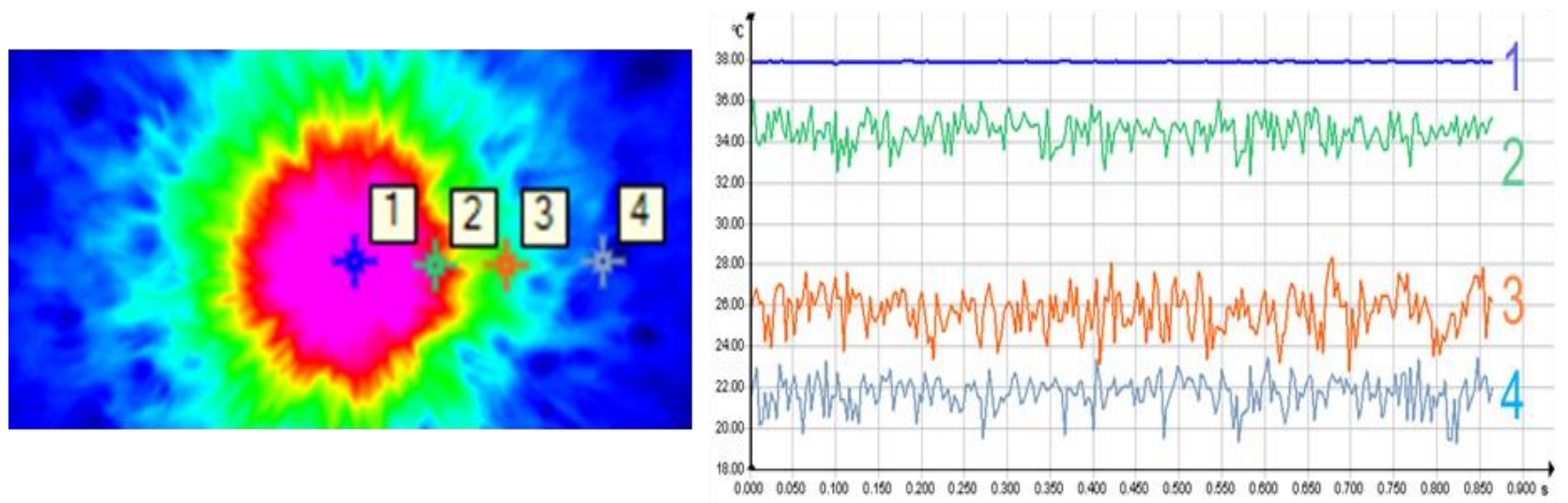

Fig. 1. A frame of thermographic animation of the impact jet and time evolution of the temperature at four points (Altair software).

\section{High-speed shadowgraph imaging}

The application of modern optical high-speed digital cameras allows studying high-speed non-stationary processes (including supersonic flows) at shooting frequency of more than a million frames per second. Fig. 2 shows 4 shadowgraph frames of the water jet formation process: visualization at the top area motion. Time interval between frames $0.03 \mathrm{~ms}$ is enough for speed measurement. 

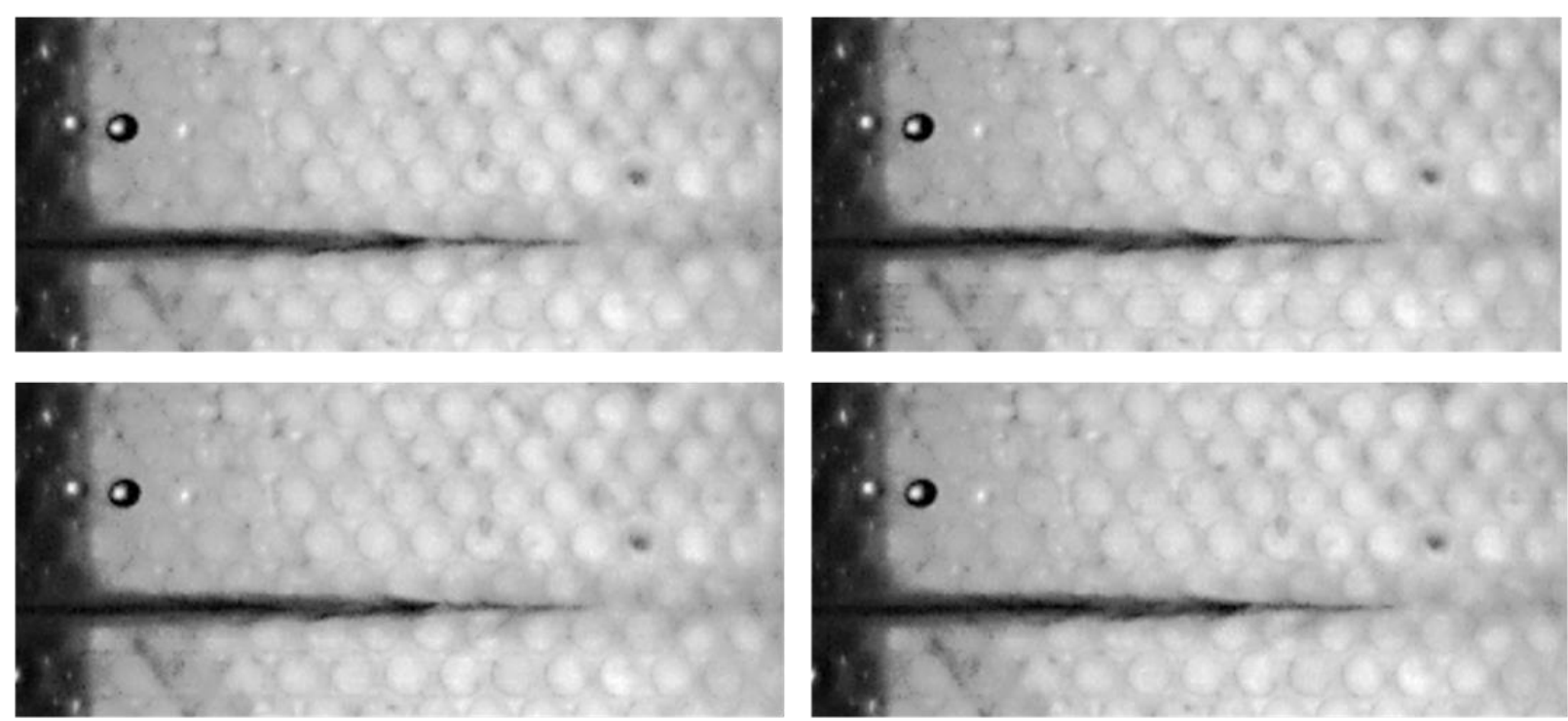

Fig. 2. Shadowgraph of water jet images captured by high-speed camera

(light absorption) 100000 frames/s

The leader speed (measured manually) increases from 30 to $250 \mathrm{~m} / \mathrm{s}$.

Fig. 3 shows 4 consequent shadowgraph images of 2 shock waves initiated by a nanosecond discharges when recording with a high-speed digital camera (124 000 frames/s). Cylindrical shock (blast) wave interacts with plane shock here.
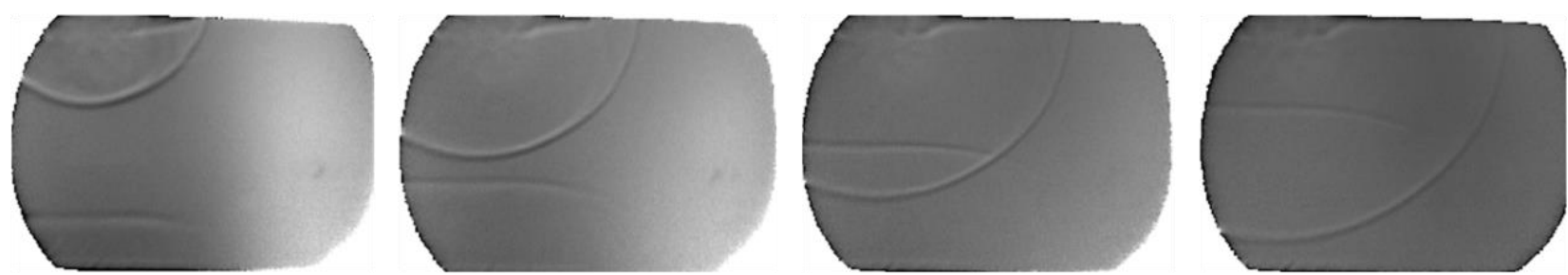

Fig. 3. Shadowgraph images (light refraction) captured by high-speed camera at 124000 frames/s

Supersonic gas flow structure can be visualized by the electrical discharge [5]. Discharge plasma in gas volume follows shapes of discontinuities, inhomogeneities, streamlines because discharge electric current strongly depends on the E/N ratio [5, 6]. Fig. 4 presents 9 frames of discharge glow zone: ionization upon interaction of a shock wave with a pulse volume discharge in the nanosecond range. Images are taken with high-speed CCD camera connected to PC. Animation of 9 frames is assembled to study the dynamics of nanosecond-lasting plasma configuration evolution. 


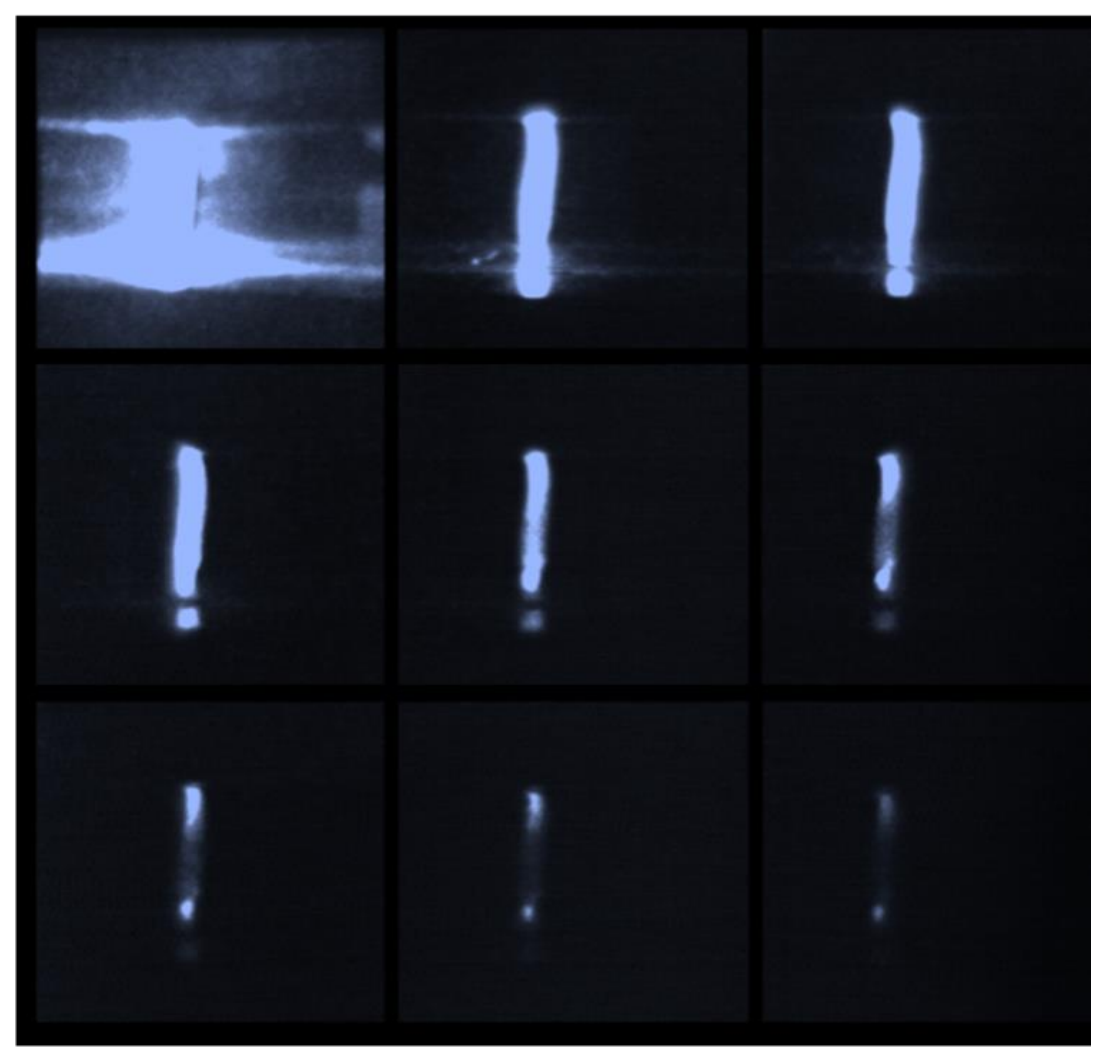

Fig. 4. Images of discharge glow captured by high-speed camera; 100 ns exposure; 100 ns time interval between frames.

\section{Computer vision and machine learning}

Applications employing machine learning in geophysics, oil, gas technologies enable today's computers to quickly and accurately analyze huge amounts of appropriate data. Large volumes of disparate kinds of oil and gas data are analyzed by machine learning algorithms to discover unknown relationships, those that were not identified previously.

The actual problem of a panoramic digital experiment in fluid dynamics is animation data analysis that is a big data analysis problem. In the experimental fluid dynamics today a huge amount of digital information is accumulated, obtained during video filming with digital cameras of different types, thermal imagers, etc. The development of digital technologies leads to a multiple increase in the data array of the gas dynamical, thermophysical flow fields parameters. The obtained large arrays of digital data often can not be processed manually. Thus, modern video films recording the evolution of turbulent fluid flows based on shadow methods, tracing, thermography require processing and qualified analysis. The transition to another level of data analysis is predictable in fluid dynamics.

When working with big data, machine learning may help analyzing large data arrays (in our case, flow images) [7]. So far, very few papers have been devoted to this problem, but their number is growing rapidly. Neural networks can effectively capture gas flow structures on large datasets [8], predict [9] and reconstruct [10] flow development using Image Retrieval, Template Matching, Parameters Regression, Spatiotemporal Prediction and other techniques. Deep learning may be used to model high-dimensional gas-dynamic systems such as turbulence [11]. Image classification and object detection systems are being developed, for example, for shock wave detection [8], bow-shock refraction angle tracking [12] or vortex wakes detection and classification [13].

Different computer vision algorithms are also applied for processing of digital animations of flows. The most used methods are edge detection, background image subtraction, noise removal [14].

In the present study the two software tools were made for flow structures automatic detection and tracking. The first tool is our in-house code for shock wave detection based on 
the modified Canny edge detection and Hough transform algorithms $[15,16]$. Edge detection is used to represent possible shock wave boundaries and the Hough transform is used to find boundaries close to the straight line. Also, we apply some line length and angle filters in our code and combining close short lines into one.

Fig. 5 shows an example of shadowgraph image processing using our software: oblique shock detection and automatic angle calculation. The entire video contains several hundred frames. The oblique shock was created by a small obstacle placed on the bottom wall of the shock tube channel.
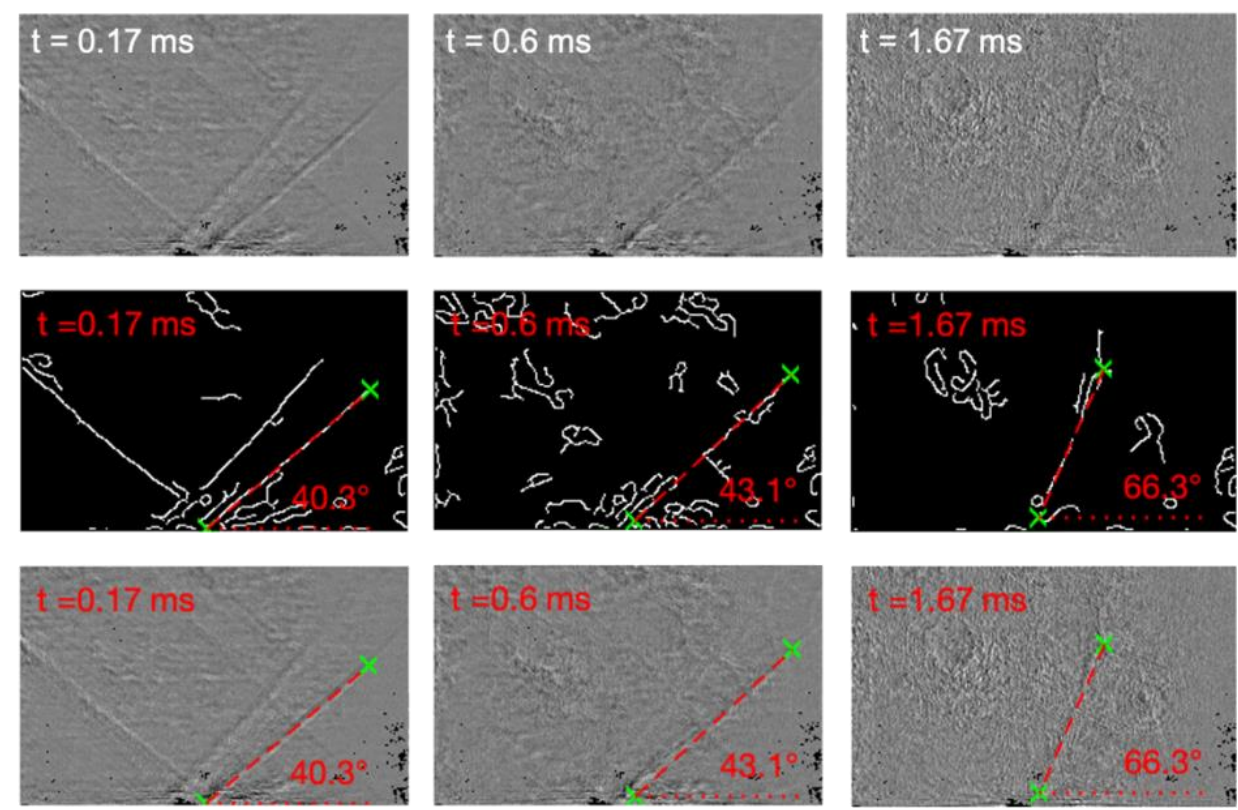

Fig. 5. Automatic image processing: applying edge detection and Hough transform to detect oblique shock and calculate its angle; first row - source images; second row - edge and line detection; third row - source image with the detected oblique shock and calculated angle.

The second software tool was developed using the convolutional neural network based on the well-known YOLOv2 architecture. The network was trained to detect three classes of objects on the images: shock waves, convective plumes, and tracer particles. We used up to 800 images for training. Some of them are featured in our online gallery [17]. The detailed information about the software is given in $[15,16,18]$. Fig. 6 shows example frames of the post-discharge thermal plume development and its automatic detection by the neural network. The full animation contains several thousand images.
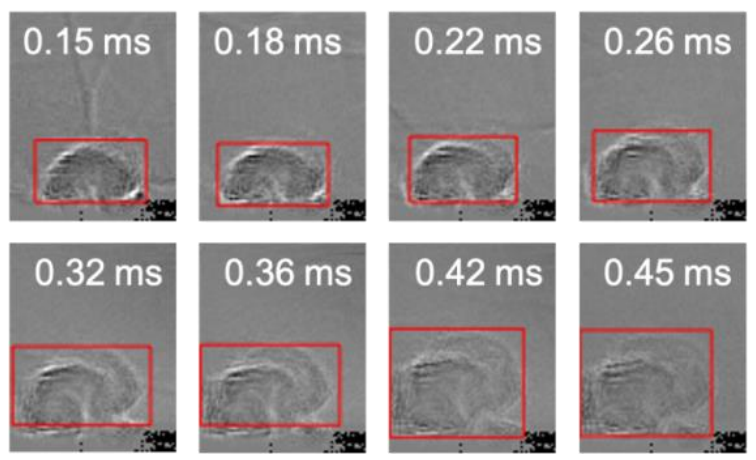

Fig. 6. Automatic image processing: thermal plume detection using convolutional neural network

Also, we made specific in-house code for bow-shock position tracking based on the pixel intensity analysis. We processed shadowgraph image sequence with bow-shock wave near the model in the flow with $\mathrm{M} \approx 2$. The experiment description is given in [19]. The image 
processing algorithm includes brightness averaging along a given band (Fig. 7, a), mean brightness local extrema detection and search for the local extrema pattern corresponding to the bow shock based on the distance between the local extrema on the intensity-distance plane (Fig. 7, b).

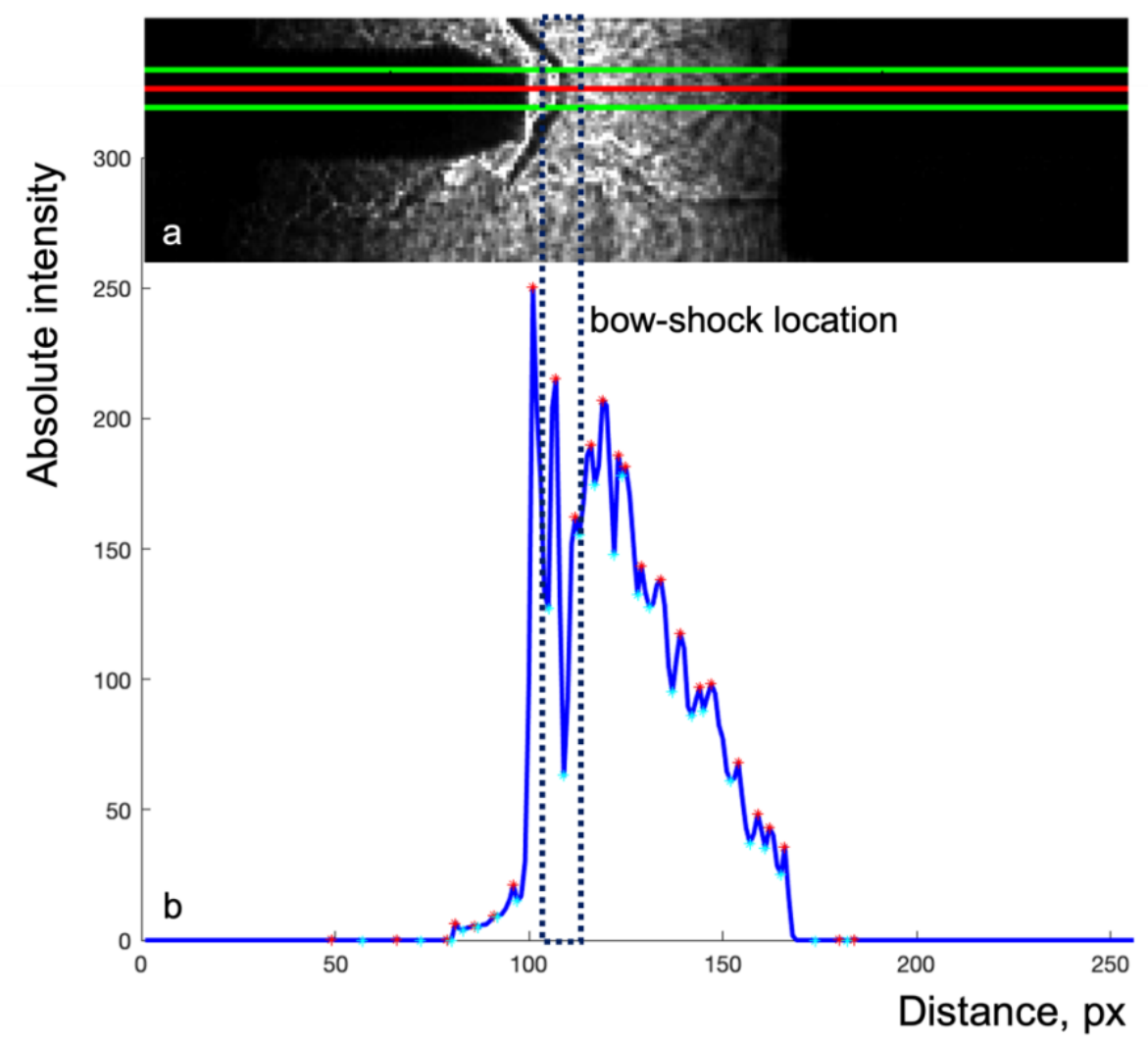

Fig. 7. Bow shock detection based on the light intensity distribution analysis

Fig. 8 shows sequence of images with automatic bow shock detection. The recording frame rate was 100 ooo frames/s. Vertical line indicates detected bow-shock position. It increases from frame 1 to 40 and then oscillates near the constant value due to the flow turbulent pulsations.
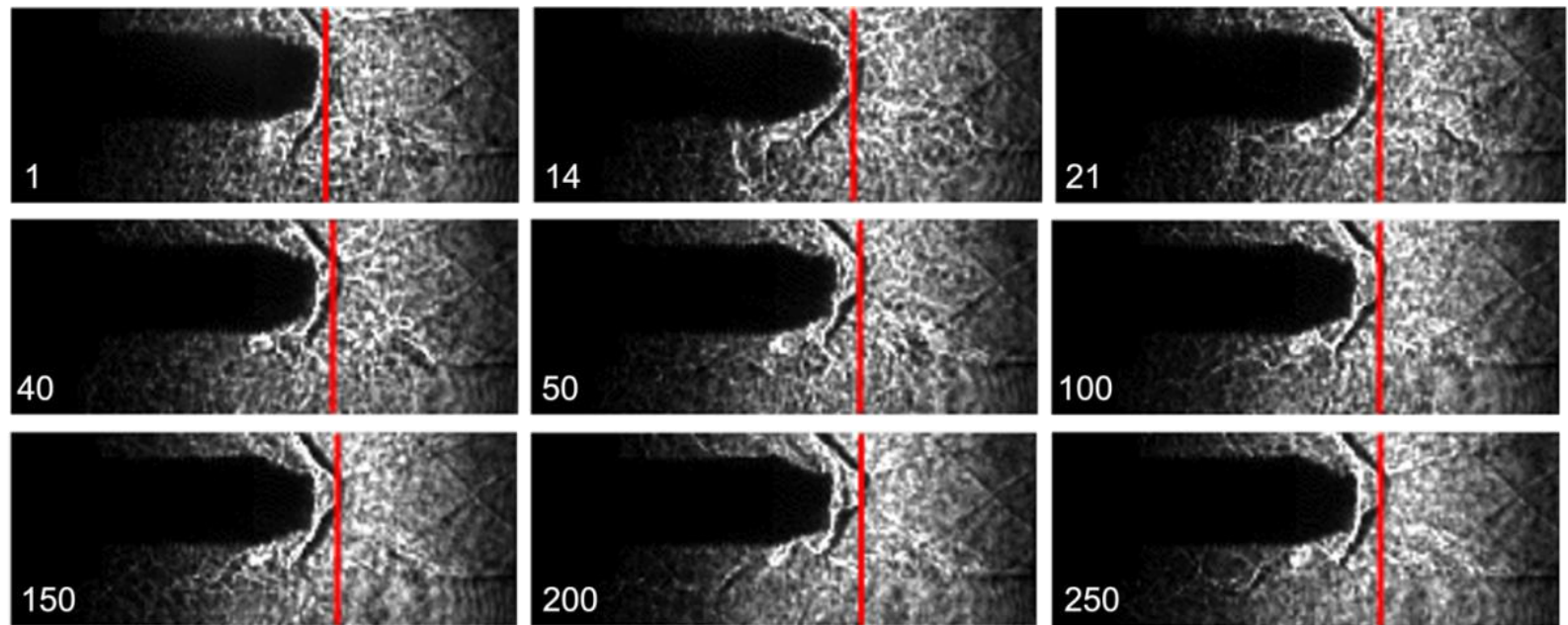

Fig. 8. Bow shock tracking image sequence. The number on images is a frame number

Distance versus time (frame number) dependency was obtained (see Fig. 9). The distance was measured starting from the left boundary of the image. It allows us to study bow shock 
stand-off distance from the model. The distance strongly depends on the flow velocity and its turbulent pulsations.

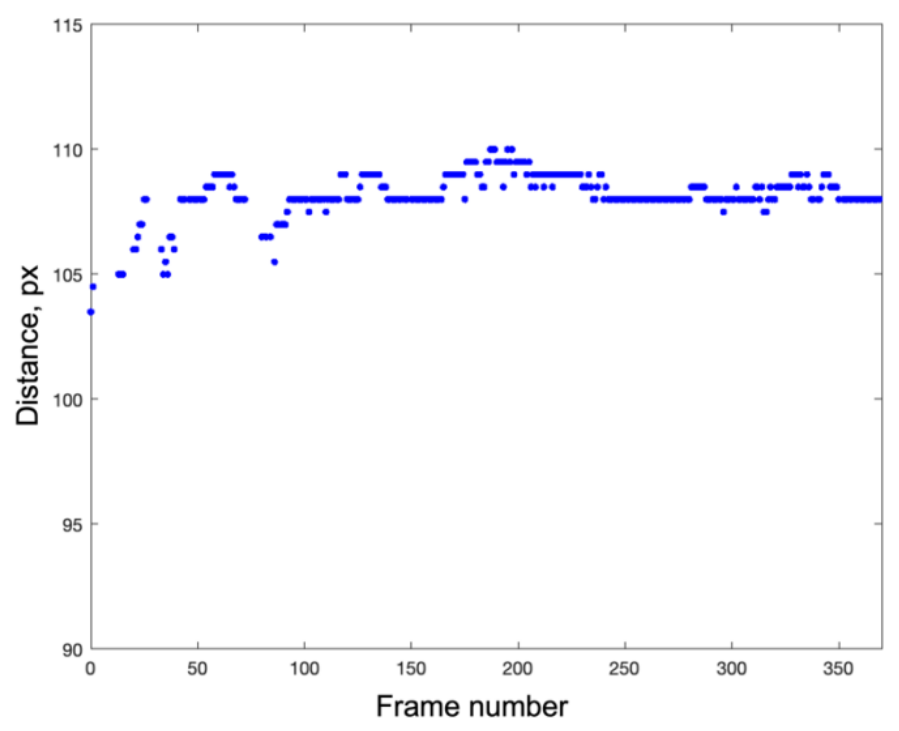

Fig. 9. Bow shock position versus frame number (time) dependency

\section{Conclusion}

Digital scientific animation is one of the main tools for studying non-stationary flows. Modern high-speed cameras support video recording at a frame rate of up to 1000000 frames/s which makes it possible to study high-speed processes. Digital animations may match CFD calculations to validate the CFD code and improve its accuracy. Some examples one can find in review [20]. Here we presented example of temperature evolution from thermographic animations (IR radiation emission) obtained at $115 \mathrm{~Hz}$ to measure fluid temperature with a high frame rate in different image points using commercial software. Also, we analyzed high-speed shadowgraph images (based on refraction) of different high speed gas flows. Supersonic water jet formation process was recorded at a frame rate of 100 ooo frames/s (example of light absorption). Shadowgraph animations of the shock waves created by the pulsed sliding discharges were recorded at 124 ooo frames/s (light refraction example). Also, we present 9 sequential images obtained by the special high-speed CCD camera with the 100 ns delay between frames (plasma radiation emission). Such short time intervals are suitable for pulsed electrical discharge visualization, it can be presented as short animation as well.

Thus, flow animations obtained with different methods of electromagnetic radiation recording give a lot of digital visual information about gas dynamics and thermophysics. To study big data of shock waves evolution (bow shock and oblique shock in the channel, as well as flow behind the shock initiated by linear discharge - the thermal plume) we made two software for automatic flow structures detection and tracking, based on the machine vision and learning techniques for automatic animations processing.

The first tool is our in-house code for shock wave detection based on the modified Canny edge detection and Hough transform algorithms. The second software tool was developed using the convolutional neural network based on the YOLOv2 architecture.

New quantitative information was obtained on bow shock position evolution in time interval 3-4 ms and also oblique shock angle changing was calculated. 


\section{Acknowledgements}

This study was carried out within the framework of the Development Program of the Interdisciplinary Scientific and Educational School of Moscow State University "Photonic and Quantum Technologies: Digital Medicine.”

\section{References}

[1] Emelyanov V. N., Volkov K. N. Visualization of physical and mathematical modeling data in gas dynamics // Moscow: Fizmatlit, 360 p., 2018, ISBN: 978-5-9221-1774-6.

[2] Settles G. S. Schlieren and Shadowgraph Techniques: Visualizing Phenomena in Transparent Media // Springer, 2001. ISBN: 978-3-642-56640-0.

[3] Znamenskaya I. A., Koroteeva E. Yu., Shirshov Ya. N., Novinskaya A. M., Sysoev N. N. High speed imaging of a supersonic waterjet flow // Quantitative InfraRed Thermography Journal, Vol. 14, № 2, 2017, pp. 185-192 (doi: 10.1080/17686733.2016.1243749).

[4] Bolshukhin M. A., Znamenskaya I. A., Fomichev V. I. A method of quantitative analysis of rapid thermal processes through vessel walls under nonisothermal liquid flow // Dokl. Phys., Vol. 6o, 2015, pp. 524-527 (doi: 10.1134/S1028335815110014).

[5] Nishio M., Sezaki S., Nakamura H. Visualization of flow structure around a hypersonic re-entry capsule using the electrical discharge method // Journal of Visualization, Vol. 7, 2004, pp. 151-158 (doi: 10.1007/BFo3181588).

[6] Znamenskaya I. A., Koroteev D. A., Popov N. A. A nanosecond high-current discharge in a supersonic gas flow // High Temperature, Vol. 43, 2005, pp. 817-824 (doi: 10.1007/s10740-005-0129-x).

[7] Brunton S. L., Noack B. R., Koumoutsakos P. Machine Learning for Fluid Mechanics // Annual Review of Fluid Mechanics, Vol. 52, 2020, pp. 477-508 (doi: 10.1146/annurevfluid-010719-060214).

[8] Monfort M., Luciani T., Komperda J., Ziebart B., Mashayek F., Marai G. E. A Deep Learning Approach to Identifying Shock Locations in Turbulent Combustion Tensor Fields // Modeling, Analysis, and Visualization of Anisotropy, 2017, pp. 375-392 (doi: 10.1007/978-3319-61358-1_16).

[9] Harel R., Rusanovsky M., Fridman Y., Shimony A. and Oren G. Complete Deep Computer-Vision Methodology for Investigating Hydrodynamic Instabilities // In: Jagode H., Anzt H., Juckeland G., Ltaief H. (eds) High Performance Computing. ISC High Performance, 2020. Lecture Notes in Computer Science, Vol. 12321, 2020, pp. 61-80 (doi: 10.1007/978-3030-59851-8_5).

[10] Ott C., Pivot C., Dubois P., Gallas Q., Delva J., Lippert M., Keirsbulck L. Pulsed jet phase-averaged flow field estimation based on neural network approach // Experiments in Fluids, Vol. 62, № 79, 2021 (doi: 10.1007/so0348-021-03180-0).

[11] Kutz J. Deep learning in fluid dynamics, Journal of Fluid Mechanics // Vol. 814, 2017, pp. 1-4 (doi: 10.1017/jfm.2016.803).

[12] Dehghan Manshadi M., Vahdat-Nejad H., Kazemi-Esfeh M. and Alavi M. Speed Detection in Wind-tunnels by Processing Schlieren Images // IJE TRANSACTIONS A: Basics Vol. 29 № 7, 2016, pp. 962-967 (doi: 10.5829/idosi.ije.2016.29.07a.11).

[13] Colvert B., Alsalman M., Kanso E. Classifying vortex wakes using neural networks // Bioinspiration \& Biomimetics, Vol. 13, № 2, 2018 (doi: 10.1088/1748-3190/aaa787).

[14] Li G., Burak Agir M., Kontis K., Ukai K. and Rengarajan S. Image Processing Techniques for Shock Wave Detection and Tracking in High Speed Schlieren and Shadowgraph Systems // Journal of Physics: Conference Series, Vol. 1215, 2019 (doi: 10.1088/1742-6596/1215/1/012021).

[15] Znamenskaya I. A., Doroshchenko I. A. Edge detection and machine learning for automatic flow structures detection and tracking on schlieren and shadowgraph images // Journal of Flow Visualization and Image Processing, Vol. 28 № 4, 2021, pp. 1-26 (doi: 10.1615/JFlowVisImageProc.2021037690). 
[16] Znamenskaya I., Doroshchenko I., Tatarenkova D. Edge Detection and Machine Learning Approach to Identify Flow Structures on Schlieren and Shadowgraph Images // CEUR Workshop Proceedings, Vol. 2744, 2020, pp. 1-14 (doi: 10.51130/graphicon-2020-2-315).

[17] Gallery of Photos and Videos, 2021. URL: http://molphys.phys.msu.ru/galery.

[18] Znamenskaya I., Doroshchenko I., Sysoev N. Edge detection and machine learning application for shadowgraph and schlieren images analysis // Proceedings of the 19th International Symposium on Flow Visualization, Shanghai Jiaotong University Press, 2021, pp. 121-130.

[19] Znamenskaya I. A., Naumov D. S., Sysoev N. N., Chernikov V. A., Analysis of Dynamic Processes Occurring during Generation of Plasmoid Formations in a Supersonic Flow // Technical Physics, Vol. 64, № 6, 2019, pp. 802-806 (doi: 10.1134/S1063784219060252).

[20] Znamenskaya I. A. Methods for Panoramic Visualization and Digital Analysis of Thermophysical Flow Fields. A Review // Scientific Visualization, Vol. 13, № 3, 2021, pp. 125 - 158, (doi: 10.26583/sv.13.3.13). 